\title{
Single-polarization, dual-wavelength mode-locked Yb-doped fiber laser by a $45^{\circ}$-tilted fiber grating
}

\author{
Xianglian Liu ${ }^{1 *}$, Hushan Wang², Yishan Wang ${ }^{2}$, Zhijun Yan ${ }^{3}$, Lin Zhang ${ }^{3}$, \\ 1. Key laboratory of Advanced Transducers and Intelligent Control system, Ministry \\ of Education, College of Physics and Optoelectronics, Taiyuan University of \\ Technology, Taiyuan 030024, China \\ 2. State Key Laboratory of Transient Optics and Photonics, Xi'an Institute of Optics \\ and Precision Mechanics, Chinese Academy of Sciences, Xi'an 710119, China \\ 3. The Photonics Research Group, Aston University, Birmingham, B4 7ET, U.K.
}

E-mail: 1xlfeimi@163.com

\begin{abstract}
We experimentally demonstrate an all-fiber single-polarization dual-wavelength $\mathrm{Yb}$-doped fiber laser passively mode-locked with a $45^{\circ}$-tilted fiber grating for the first time. Stable dual-wavelength operation exhibits double-rectangular spectral profile centered at $1033 \mathrm{~nm}$ and $1053 \mathrm{~nm}$, respectively. The 3-dB bandwidth of each rectangular optical spectrum is estimated as $10 \mathrm{~nm}$. The separation of two fundamental repetition rates is $6 \mathrm{kHz}$. By employing the $45^{\circ} \mathrm{TFG}$ with the polarization-dependent loss of $33 \mathrm{~dB}$, output pulses with 27-dB polarization extinction ratio are implemented in the experiment. The single pulse centered at 1053 $\mathrm{nm}$ is researched by using a filter at the output port of laser, and the experimental results denote the output ps pulses are highly chirped. The formation mechanism of dual-wavelength operation is investigated.

Keywords: Fiber lasers, single-polarization, dual-wavelength, fiber grating
\end{abstract}

PACS: 42.55.Wd; 42.65.Tg; 42.81.Dp

\section{Introduction}


Passively mode-locked fiber lasers have attracted considerable interest and been extensively investigated in the past two decades for their compact configuration, low thermal effect, and good flexibility [1-8]. Thereinto, multi-wavelength fiber lasers have the potential for applications in many fields such as optical instrumentation, wavelength division multiplexing (WDM) networks, testing of WDM components, optical sensing, and photonic component characterization. For the erbium-doped fiber laser, several methods for implementing the multi-wavelength operation have been researched [9-13].

Recently, multi-wavelength Yb-doped fiber (YDF) lasers with different configurations and mechanisms for the wavelength selection and stabilization have been reported. These techniques include dual wavelength operation using a fiber Bragg grating (FBG) stabilized by four wave mixing [14], the use of spatial mode beating in multimode fiber [15], dual frequency generation using the birefringence of a polarization-maintaining FBG [16], a few-mode FBG together with polarization controller (PC) working as a filter [17], and a Mach-Zehnder interferometer operating as a comb filter [18]. However, these multi-wavelength YDF lasers emit continuous wave (CW). Up to now, the researches for multi-wavelength pulsed YDF lasers are relatively rare [19-21]. Pan et al. have realized a passively Q-switched dual-wavelength Yb fiber laser using a Cr4+:YAG saturable absorber [19]. Hu et al. have reported a dual-wavelength ns pulse in an Yb-doped fiber laser based on the stimulated Brillouin scattering [20]. Zhu et al. have demonstrated a stable and switchable dual-wavelength mode-locked operation by using a phase-shifted long-period fiber grating as a spectral filter [21]. The aforementioned setups sacrifice the feature of all-fiber configuration. The all-fiber format without coupling between fiber and bulk segments has great stability, compact design and low insertion loss, so, an intense effort is now carried out to implement the all-fiber laser. A $45^{\circ}$-tilted fiber grating (TFG) is exploited to realize the mode locking for implementing the all-fiber configuration.

To the best of my knowledge, we report for the first time on a single-polarization all-fiber, dual-wavelength, Yb-doped fiber laser passively mode-locked by a $45^{\circ}$ TFG. The $45^{\circ}$ TFGs that can discard the s-polarization component from fiber core and leave the p-polarization component propagating in the fiber core with minimum loss is used as a polarizer in the passively mode-locked fiber laser. The polarization-dependent loss (PDL) of $45^{\circ} \mathrm{TFG}$ used in 
the experimental setup is $33 \mathrm{~dB}$. When the pump power increases to $339 \mathrm{~mW}$ at the appropriate PC settings, stable dual-wavelegnth mode-locking ps pulses centered at the 1033 $\mathrm{nm}$ and $1053 \mathrm{~nm}$ are simultaneously achieved. The 3-dB bandwidth of each rectangular spectral profile is almost equivalent, which is evaluated as $\sim 10 \mathrm{~nm}$. The peak to pedestal extinction is greater than $65 \mathrm{~dB}$. By exploiting the 10-nm band-pass filter centered at $1053 \mathrm{~nm}$, the characteristic of single pulse is investigated. Adjusting the polarization controller (PC) outside of the laser cavity, the polarization extinction ratio (PER) of output pulses is measured as $\sim 27 \mathrm{~dB}$, which indicates that the pulse is linearly polarized. In general, the commercial fiber polarization dependent isolator only has $25-\mathrm{dB}$ PER, therefore, the $45^{\circ}$ TFGs have superiority of obtaining single-polarization passively mode-locked pulses. According to the experimental results, we attribute the formation of dual-wavelength pulses to the combined effects of linear fiber birefringence and fiber nonlinearity.

\section{Experimental setup}

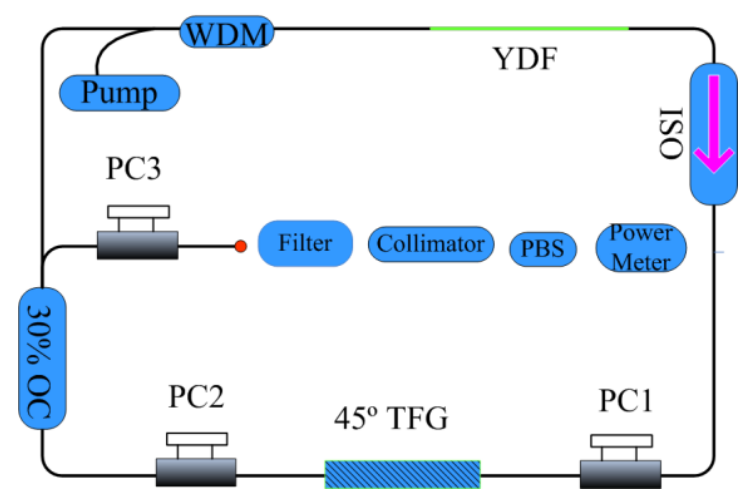

Fig. 1 Experimental setup of the fiber ring laser.

The $45^{\circ}$ TFG is UV-inscribed using the phase-mask scanning technique and a frequency-doubled Ar laser. Fabrication and measuring PDL of $45^{\circ}$ TFG have been described in detail in reference 22. The characterization of $45^{\circ}$ tilted fiber grating used in this experiment has been described in detail in reference 23 . The $45^{\circ} \mathrm{TFG}$ with $33-\mathrm{dB}$ PDL is used as a polarizer to implement the mode locking. The configuration of the fiber laser is schematically shown in Fig. 1. It consists of a segment of $45^{\circ}$ TFG, a 980/1053 nm wavelength-division-multiplexed (WDM) coupler, a 0.73-m long YDF with absorption coefficient of $500 \mathrm{~dB} / \mathrm{m}$ at $976 \mathrm{~nm}$, a polarization insensitive isolator (PI-ISO), a fused optical coupler (OC) with $30 \%$ output, two set of PCs, and 5.57-m standard SMF. The dispersion 
parameters for YDF and SMF are $20 \mathrm{ps}^{2} / \mathrm{km}$ and $22.1 \mathrm{ps}^{2} / \mathrm{km}$ at $1050 \mathrm{~nm}$, respectively. The net dispersion of the cavity is calculated as $0.14 \mathrm{ps}^{2}$. A $980 \mathrm{~nm}$ laser diode is used to provide the pump. An ISO is used to make the light propagate unidirectionally in the cavity and promote the pulse formation. The single pulse is further studied by using a $10-\mathrm{nm}$ filter centered at $1053 \mathrm{~nm}$ at the output port of laser. The output properties of the laser oscillator are monitored by a $10 \mathrm{GHz}$ photodetector (PD), an optical spectrum analyzer, a digital storage oscilloscope (DSO) with 6-GHz-bandwidth, a radio-frequency (RF) analyzer, a power meter, and a commercial autocorrelator. The polarization state of output pulse is further studied by employing a bulk polarization beam splitter (PBS) with 40-dB PER and a PC.

\section{Experimental results and discussions}
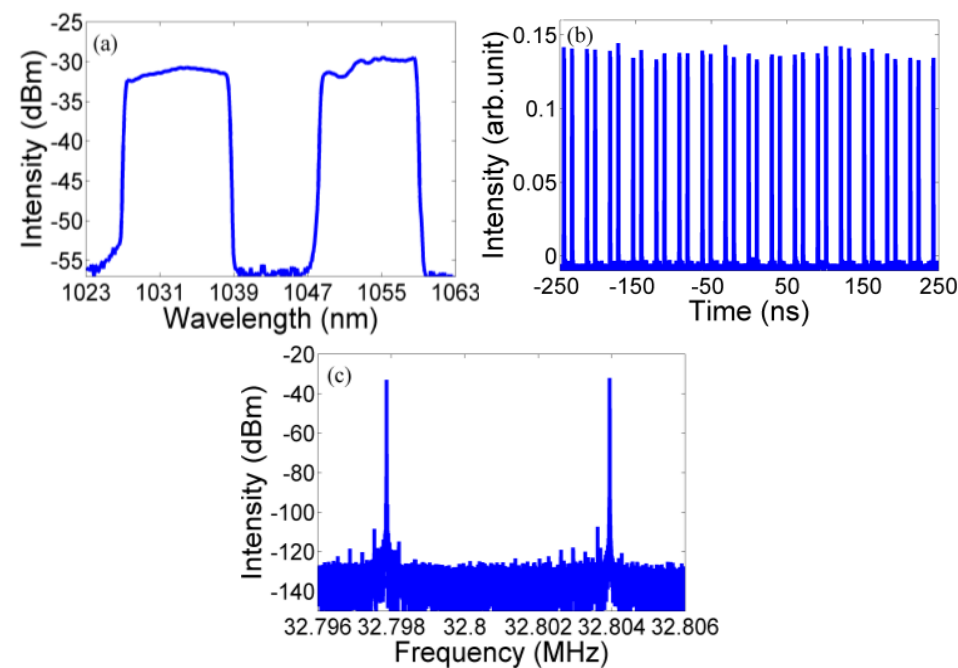

Fig. 2 Optical spectrum (a), oscilloscope trace (b), and RF spectrum (c) at dual-wavelength operation when the pump power is $339 \mathrm{~mW}$.

According to Fresnel's law, the $45^{\circ}$ TFGs couple out the s-polarization component of light and leave the p-polarization component travelling in the fiber [22]. Consequently, the $45^{\circ} \mathrm{TFG}$ used as a polarizer and the two PCs are exploited for the stable self-started passive mode locking. With appropriately adjusting two PCs when the pump power is increased above the mode-locked threshold, the dual-wavelength mode-locking state can be easily realized. The dispersion of the laser cavity is normal, thus, the dissipative solitons (DS) operation can be obtained. As shown in Fig. 2(a), stable dual-wavelength DSs at the pump of $339 \mathrm{~mW}$ are simultaneously obtained. The generated pulses exhibit the typical spectral profile of DSs with 
steep edges and the $3-\mathrm{dB}$ bandwidth of rectangular optical spectra centered at $1033 \mathrm{~nm}$ and $1053 \mathrm{~nm}$ is evaluated as $\sim 10 \mathrm{~nm}$, respectively. Figure 2(b) shows dual-wavelength mode-locked pulses train measured with the PD and DSO at a given time, which indicates that two individual and temporally separated mode-locked pulses are formed in the laser cavity. The two uniform pulse heights denote that the corresponding energy of two pulses is nearly identical, which is other than the two different pulse heights in the oscilloscope trace as reported in reference 9. The result is described as soliton energy quantization. The pulse train is distinct from the ordinary single-pulse or single-wavelength dual-pulse train. For the single-pulse mode locking, the pulse-to-pulse separation is fixed, which is in accordance with the time of circulating one trip. For the single-wavelength dual-pulse mode locking, the two pulses have fixed separation circulating in the laser cavity whereas the pulse separation is smaller than the time of circling one trip. However, for the dual-wavelength mode locking, each pulse has the different velocity and the round-trip time because of dispersive effect. Consequently, the pulse-to-pulse separation is not a fixed value. In the experiment, we trigger one pulse and then the other pulse moves randomly on the oscilloscope screen. Further checking the pulse with the PD and RF analyzer, the RF spectrum of dual-wavelength DSs is depicted in Fig. 2(c). The dual-wavelength operation exhibits two fundamental repetition rates with 6-kHz gap, which is different from the single-wavelength mode-locked operation with only one fundamental repetition rate. The fundamental repetition rate of the laser cavity is direct proportional to the velocity of pulse, thus, different repetition rate is corresponding different velocity. It is further confirmed that the two mode-locked pulses at different wavelengths are not synchronized in temporal region. The results of radio-frequency spectrum are in good agreement with the pulse train. The peak to pedestal extinction at a resolution bandwidth of $1 \mathrm{~Hz}$ is higher than $65 \mathrm{~dB}$. The average output power of two pulses is $31 \mathrm{~mW}$. The polarization state of the dual-wavelength DSs is investigated by employing a bulk PBS and a PC3, as shown in Fig. 1. The bulk PBS with 40 dB PER is used to resolve the two orthogonally polarized components. The PC3 modulates the linear birefringence of fiber to change the polarization state of the dual-wavelength DSs. The power meter measures the power from one output port of the PBS. Adjusting the PC3, the PER of output pulses is calculated as $\sim 27 \mathrm{~dB}$ by measuring the maximum and minimum output power, which 
indicates the pulses are nearly totally polarized.
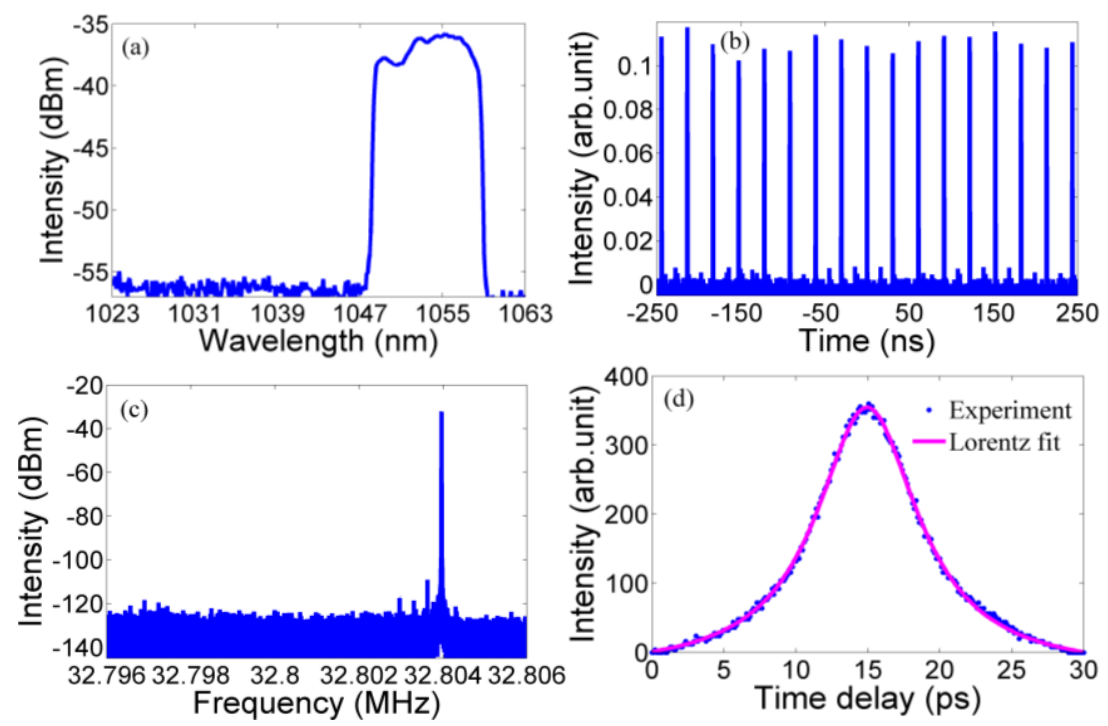

Fig. 3 Optical spectrum (a), oscilloscope trace (b), RF spectrum (c), and autocorrelation trace (d) at dual-wavelength operation when the pump power is $339 \mathrm{~mW}$ with $10-\mathrm{nm}$ band-pass filter at $1053 \mathrm{~nm}$ used on the output port of the fiber laser.

In order to separate each pulse, a 10-nm band-pass filter centered at $1053 \mathrm{~nm}$ is used at the output port of the fiber laser, which is described in Fig. 1. Compared with the Fig. 2(a), the optical spectrum centered at $1033 \mathrm{~nm}$ is eliminated by the band-pass filter as shown in Fig. 3(a). The 3-dB width of output spectrum is evaluated as $\sim 10 \mathrm{~nm}$. Figure 3(b) exhibits that the pulse train is fixed on the oscilloscope screen and quite uniform with pulse-pulse separation of $\sim 30.5 \mathrm{~ns}$, which can confirm the output pulses centered at $1053 \mathrm{~nm}$ maintain stable single-pulse mode-locking operation. The quite uniform of pulse sequence is attributed to the soliton energy quantization effect. The experimental results fully indicate that the two pulses centered at $1033 \mathrm{~nm}$ and $1053 \mathrm{~nm}$ are essentially non-interacting. The group index of fused silica is in the inverse ratio to wavelength and hence the fundamental repetition rate for the long-wavelength pulse is larger than that of short-wavelength pulse. As displayed in Fig. 3(c), the large fundamental repetition rate is retained alone, which is also in excellent agreement with the above analysis. Compared with Fig. 2(c), we draw a conclusion that the pulses centered at $1033 \mathrm{~nm}$ and $1053 \mathrm{~nm}$ have the $32.798-\mathrm{MHz}$ and $32.804-\mathrm{MHz}$ fundamental repetition rate of the laser cavity, respectively. The autocorrelation trace takes on a Lorentz 
shape, and the pulse width is given by $\sim 4.3$ ps (Fig. 3(d)). Compared with the previous reports [19-21], the obtained the pulse duration is relatively short. The corresponding time-bandwidth product of the pulse is estimated as 12.5 , indicating that the pulse is highly chirped. The average output power of pulse centered at $1053 \mathrm{~nm}$ is $15 \mathrm{~mW}$, corresponding to pulse energy of $\sim 0.45 \mathrm{~nJ}$.

Remaining the pump power, by adjusting the PCs with appropriate orientation and pressuring settings, single pulse emission displacing dual-wavelength operation can be easily achieved. These experimental results indicate that the fiber birefringence plays an important role in the formation of dual-wavelength mode locking. Based on the linear transmission model, the cavity transmission of the fiber laser versus wavelength is a sinusoidal function [27, 29-31]. The dual-wavelength operation tends to establish at the adjacent transmission maxima of a birefringence filter. At the proper experimental condition, the shorter and longer wavelengths experience lower loss, while the mid-wavelength undergoes much higher loss. Under pump, the shorter and longer wavelengths are amplified, while the mid-wavelength is suppressed. As a result, the dual-wavelength mode locking will be obtained as the power becomes large enough. References 9-13, 24-28 have indicated that the linear fiber birefringence plays a key role in the formation of dual-wavelength mode locking.

We further investigate the dual-wavelength pulses evolution as a function of pump power. Slowly increasing the pump power while maintaining the other cavity parameters, the dual-wavelength operation is still in stable state until the pump power is beyond $389 \mathrm{~mW}$. When the pump power is $389 \mathrm{~mW}$, the region of shorter wavelength starts to become unstable. If the pump power is further increased, the mode locking of shorter wavelength becomes more and more unstable until the state of mode locking is destroyed and the $\mathrm{CW}$ laser emission could be observed (shown as in Fig. 4(a)). At the $444 \mathrm{~mW}$ pump power, the CW emission of shorter wavelength dismisses and the fiber laser operates in stable single-long-wavelength mode locking (shown as in Fig. 4(b)). On the contrary, decreasing the pump power, the stable single-short-wavelength mode locking can be observed. The pulse evolution with the pump power, which is attributed to the transmission coefficient of the laser cavity changed with the power. 

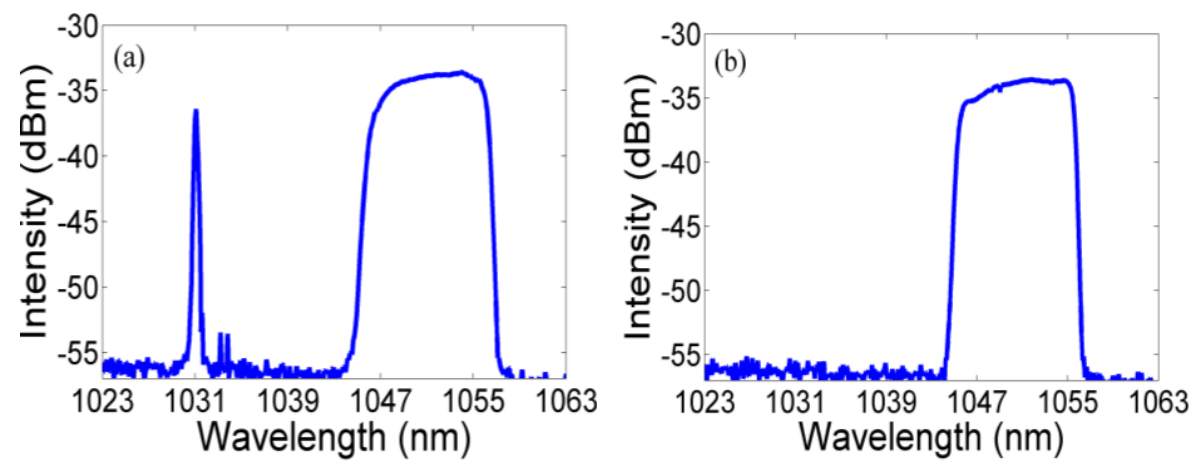

Fig. 4 Optical spectra at $435 \mathrm{~mW}$ (a) and $444 \mathrm{~mW}$ (b) pump power.

Thus, the fiber laser delivers the stable dual-wavelength pulses due to the combined effects of the linear fiber birefringence and fiber nonlinearity.

\section{Conclusion}

We present to the best of our knowledge the first single-polarization all-fiber, dual-wavelength, Yb-doped passive mode-locking fiber laser. When the pump power is 339 $\mathrm{mW}$, the fiber laser emits ps pulses with the rectangular spectral profile centered at $1033 \mathrm{~nm}$ and $1053 \mathrm{~nm}$, respectively. Each rectangular optical spectrum width is evaluated as $10 \mathrm{~nm}$. The RF spectrum of dual-wavelength operation reveals the 6-kHz gap of the two fundamental repetition rates and $65-\mathrm{dB}$ peak to pedestal extinction. The output pulses with 27-dB PER, which denotes that the pulses operate in the single-polarization state. By employing a band-pass filter centered at $1053 \mathrm{~nm}$, the single pulse is investigated, and the experimental results indicate the pulses are highly chirped. Attributing to the combined effects of the linear fiber birefringence and fiber nonlinearity, this fiber laser delivers the stable dual-wavelength pulses.

\section{Acknowledgements}

The research was supported by the Qualified Personnel Foundation of Taiyuan University of Technology (Grant No. tyut-rc201387a), National Natural Science Foundation of China (Grant No. 61227016), the National Science and Technology Infrastructure Program of Science and Technology of Shanxi Province, China (Grant No. 2013091021)

\section{References}

[1] Sotor J, Sobon G, Macherzynski W and Abramski K M, 2014 Laser Phys. Lett. 11055102

[2] Choi S Y, Jeong H, Hong B H, Rotermund F and Yeom D, 2014 Laser Phys. Lett. 11 055101 
[3] Liu X M, Han D D, Sun Z P, Zeng C, Lu H, Mao D, Cui Y D and Wang F Q, 2013 Sci. Rep. 32718

[4] Nyushkov B N, Ivanenko A V, Kobtsev S M, Turitsyn S K, Mou C, Zhang L, Denisov V I, and Pivtsov V S, 2012 Laser Phys. Lett. 9 59-67

[5] Mao D, Liu X M, Sun Z P, Lu H, Han D D, Wang G X and Wang F Q, 2013 Sci. Rep. 3 3223

[6] Liu X L, Wang H S, Wang Y S, Zhao W, Zhang W, Tan X F, Yang Z, Shen D Y, Li C, Yan Z J, Zhang L and Chen G D, 2013 Laser Phys. Lett. 10095103

[7] Durán-Sánchez M, Álvarez Tamayo R I, Pottiez O, Ibarra-Escamilla B, Hernández-García

J C, Beltran-Perez G and Kuzin E A, 2015 Laser Phys. Lett. 12025102

[8] Zhang B F, Cao D X, Jiao Z X and Wang B, 2015 Laser Phys. Lett. 12015102

[9] Zhang H, Tang D Y, Wu X, and Zhao L M, 2009 Opt. Express 17 12692-12697

[10] Zhao X, Zheng Z, Liu L, Liu Y, Jiang Y X, Yang X, and Zhu J S, 2011 Opt. Express 19 $1168-1173$

[11] Mao D, Liu X M, L. Wang R, Lu H, and Duan L N, 2011 Opt. Express 19 3996-4001

[12] Yun L, Liu X M, and Mao D, 2012 Opt. Express 20 20992-20997

[13] Mao D, and Lu H, 2012 J. Opt. Soc. Am. B 29 2819-2826

[14] Chen L R, and Gu X J, 2007 Opt. Express 15 5083-5088

[15] Chi R, Lu K, and Chen S, 2003 Microwave Opt. Technol. Lett. 36 170-172

[16] Guan W, and Marciante J R, 2007 IEEE Photon. Technol. Lett. 19 261-263

[17] Feng X, Liu Y, Fu S, Yuan S, and Dong X, 2004 IEEE Photon. Technol. Lett. 16 762-764

[18] Tu C, Guo W, Li Y, Zhang S, and Lu F, 2007 Opt. Commun. 280 448-452

[19] Pan L, Utkin I, and Fedosejevs R, 2008 Opt. Express 16 11858-11870

[20] Hu S L, Yu J, Gao C Q, Wei G H, and Lü F Y, 2006 Chin. Opt. lett. 4 655-657

[21] Zhu X J, Wang C H, Liu S X, Hu D F, Wang J J, and Zhu C Y, 2011 IEEE Photon. Technol. Lett. 23 956-958

[22] Yan Z J, Mou C B, Zhou K M, Chen X F, and Zhang L, 2011 J. Lightw. Technol. 29 $2715-2724$

[23] Liu X L, Wang H S, Yan Z J, Wang Y S, Zhao W, Zhang W, Zhang L, Yang Z, Hu X H, Li X H, Shen D Y, Li C, and Chen G D, 2012 Opt. Express 20 19000-19002 
[24] Xu H W, Lei D J, Wen S, Fu X, Zhang J, Shao Y, Zhang L, Zhang H, and Fan D, 2008 Opt. Express 16 7169-7174

[25] Zhao L M, Tang D Y, Wu X, and Zhang H, 2010 Opt. Lett. 35 2756-2758

[26] Tang D Y, Zhao L M, Zhao B, and Liu A Q, 2005 Phys. Rev. A 72, 043816

[27] Dong J L, Xu W C, Luo Z C, Luo A P, Wang H Y, Cao W J, and Wang L Y, 2011 Opt.

Commun. $2845719-5722$

[28] Man W S, Tam H Y, Demokan M S, Wai P K A, and Tang D Y, 2000 J. Opt. Soc. Am. B 17 28-33 